\title{
BIM ecosystem research: What, why and how? Framing the directions for a holistic view of BIM
}

\author{
Vishal Singh \\ 'Department of Civil Engineering, Aalto University, Finland \\ \{Vishal.Singh\}@Aalto.fi
}

\begin{abstract}
This paper presents theoretical arguments for BIM ecosystem research, based on concepts of coevolution and emergence in a complex network of constituent elements: products, processes, people, technology and policies. Broader trends such as Internet of Things, data analytics, and digital manufacturing are increasingly becoming integral to BIM systems. Consequently BIM research is evolving along multiple pathways. Therefore, there is need for methodologies and approaches to analyze BIM ecosystem. Three types of ecosystem analyses are proposed: (1) BIM-Ecosystem Retrospective Analysis (BIM-ERA), to understand the ecosystem's constituents and their dependencies, based on facts and history, (2) BIM-Ecosystem Feasibility analysis (BIM-EFA), to be able to use the knowledge of the constituents and their dependencies to assess whether an intended intervention in a given ecosystem is feasible or not, and (3) BIM-Ecosystem Impact Analysis (BIM-EIA), to be able to conduct whatif studies to assess the potential short-term and long-term impact of a potential action.
\end{abstract}

Keywords: BIM, ecosystem, coevolution, impact analysis, research methods

\section{Introduction}

Building Information Modelling (BIM) has gained global attention across the industry, academia and the policy makers. This is fueling rapid growth of the BIM ecosystem, which, following [1], has been defined as the network of interacting technologies, processes, policies and organizations that collectively determine the development and evolution of BIM related products and services [2]. For example, BIM adoption in the US grew from $17 \%$ in 2007 to a remarkable $70 \%$ in 2012 [3]. During the same period several new BIM products and services have emerged in the market, while government agencies across countries such as the UK have created national BIM guidelines and policies. In such a dynamically evolving BIM ecosystem there is limited understanding of how the BIM technologies, processes, policies and organizations are mutually coevolving. These developments have opened a new multi-disciplinary research frontier around BIM, with long term implications for theory, education and practice.

In the recent years, several streams of BIM research have emerged across specific issues such as BIM adoption, product data models and standards, facilities management, 
and collaboration [4]. However, the lack of systemic understanding of the BIM ecosystem is a critical knowledge gap that needs attention. First, since BIM is viewed as a collaborative approach, the potential benefits of BIM are contingent on how the technologies, processes, policies and the organizations fit together e.g. [5]. Second, a comprehensive understanding of the systemic challenges is critical to facilitating effective BIM strategy and execution. Third, the emerging BIM research discipline requires strategic insights and directions for future research.

Therefore, this position paper aims to highlight the need for a theoretical basis to understand the patterns and implications of the coevolution in BIM ecosystem at the micro (e.g project and organization) and macro levels (e.g industry and society). A better understanding of the BIM ecosystem will improve decision making and collective growth for various stakeholders who are directly or indirectly part of the BIM ecosystem. For example, at the macro level, what are opportunities and challenges for different stakeholders in the evolving BIM ecosystem? What role can the government agencies, policy makers and regulations play in fostering a BIM ecosystem that is conducive to economic, environmental and social welfare? At the micro level, what are the dependencies across emerging technologies, processes and organizations that we need to know for effective management and implementation of BIM-based projects? Similarly, how are the macro and micro levels of BIM ecosystem mutually related? And, how are the broader technological trends such as internet of things (IoT) and digital manufacturing likely to affect the BIM ecosystem?

In addition, there are several fundamental conceptual issues that need to be resolved. For example, how do we define the boundary of the BIM ecosystem? Which technologies, which roles, what processes, and what policies, direct or indirect, should be considered part of the BIM ecosystem and which need not? Or a more fundamental question is whether we at all need to define the boundary? What are the theoretical and conceptual implications of the chosen approach and how could that impact BIM research and development, as well as decision making in practice?

More recently, a systemic view of BIM is starting to emerge in the discussions such as BIM research frameworks e.g. [6], BIM execution plans e.g. [7], and maturity matrices e.g. [8]. Along with these, a comprehensive understanding of BIM ecosystem is required, especially from the viewpoint of co-evolutionary mechanisms and dependencies across different levels of granularity, micro to macro. An endeavour to understand the systemic change in construction is not new. Previous attempts at forming a systemic view of the development of construction IT have typically taken the form of industry forecasting, review articles, policy documents, strategy reports and roadmaps e.g. $[9,10]$. Therefore, one of the key objectives of this paper is also to clarify how the proposed BIM ecosystem perspective is different to such approaches, and yet how the ecosystem perspective can also contribute to the development of maturity models, creating roadmaps and identifying strategic directions for research and practice.

\section{BIM Ecosystem: What should we aim to research and why?}

\subsection{Identifying the key constituents of the BIM ecosystem.}


The first steps is to identify the key constituents and categories, such that the key elements and their position in the ecosystem can be located. This iterative identification, mapping and clustering of the elements is a longitudinal process because the ecosystem continues to evolve. Such an effort will require a methodological approach, grounded in sound-theoretical and conceptual basis. An ontology to describe the BIM ecosystem is desirable. The ontological approach can build on related work in the literature. For example, [8] have described eight key components of a BIM maturity model, in the context of macro-level BIM adoption. A critical review of the different approaches to classifying and clustering the constituent elements of the BIM ecosystem is needed to assess their strengths and limitations. There is conspicuous absence of any methodological analysis or critical review of these classifications in the BIM literature. Critical questions need to be discussed and debated. For example, what are the structural characteristics of the ecosystem: is there a structural pattern at all? Is it hierarchical and/or nested and/or modular? What are the levels of details to consider? How do we ascertain the parity of the different concepts chosen for each level? etc.

\subsection{Identifying dependencies and rules of interaction between the elements}

The dependencies between the constituent elements need to be identified [11, 12]. These dependencies influence the patters of emergence, which can be traced from the historical data, but at the same time these dependencies may influence the emergent patterns of the future. While mapping the dependencies between these elements, once again several aspects need to be considered. For example, which relationships are constant and which of these are variable? What are the factors influencing the variable relationships, for e.g, temporality, regionality, etc? What are global and local variations in the dependencies? How can the dependencies be defined? 


\subsection{Developing methodologies for BIM ecosystem analysis}

Besides knowing the constituent elements of the BIM ecosystem, its structural characteristics, and the dependencies between the elements, we need methodologies to analyze the dynamic patterns of evolution- to understand how changes emanate and propagate through the ecosystem- to assess aspects such as stability, robustness and centrality of the ecosystem, as found in any complex network e.g. [13]. The methodological approaches can broadly focus on the following aspects:

BIM ecosystem retrospective analysis (BIM-ERA). The retrospective analysis is based on the past, and hence, there are historical data, facts and experiences to build an understanding of the BIM ecosystem. While the future evolution may or may not be dependent of the past events, lessons learnt from the past, and dependencies and patterns observed in the past, may provide useful insights into the future trends.

BIM ecosystem feasibility analysis (BIM-EFA). Once a mapping of the BIM ecosystem begins to emerge, it is desirable to be able to make informed decisions about potential actions that can be taken to bring about a desired change in the ecosystem. The feasibility analysis methods should allow assessing whether a potential action or solution is possible within the given ecosystem or not, that is, is the action or solution feasible? Constraints within the environment or dependencies with other potential actions or problems may render a solution non-feasible in the context. Such an assessment should also give insights into potential barriers to feasibility.

BIM ecosystem impact analysis (BIM-EIA). An action or solution that is feasible may have varied short-term and long-term impact on the ecosystem, some of which may be desirable and some may not. Thus, it is not sufficient to analyze the feasibility, but it is equally important to analyze the impact of the action or solution. The concept of BIMEIA is inspired by approaches such as Environmental Impact Assessment e.g. [14] commonly used in ecological domains, where systemic changes or likely change propagation and impact is thoroughly studied before a major action or solution is approved. This aspect has so far been entirely overlooked in the study of ICT or business ecosystems, both within and outside BIM research. Nonetheless, impact analysis should be considered for the following reasons:

Some actions or solutions may have high initial investment both in terms of cost, time and effort, which means they require systemic effort for implementation and execution over a longitudinal period of time. Such efforts can only be made occasionally. For example, in the context of BIM systems if an organization makes an investment to adopt new BIM applications across the teams, then the cost of the applications, training the staff, and beginning to use it effectively is a high investment decision. Once that has been committed to and reached mid or advanced stage, then moving to another set of applications across the teams in a short-term, even if they may be better than the chosen solution, will become extremely challenging.

Some actions or solutions may be irreversible in nature. For example, organizations may invest in building systemic processes and culture to support certain technologies 
and approaches. Once these actions and steps have been taken they cannot be undone, and more systemic effort will be required to replace them if they do not provide the intended outcomes, or worse, lead to undesirable outcomes.

While some actions or solutions may require one-off effort, other actions or solutions may require continuous effort over a longer period of time. It is important to assess whether continuous effort is sustainable or not.

In considering impact analysis, it is also important to define a benchmark to identify what is the alterative? That is, while it is important to assess what may be the impact of an action, it is also important to assess what will happen if the action is not taken? Is there an alternative action, and how does it compare? Contingent on the type of action or solution, the future course of actions such as flexibility, adaptability, scalability, etc can be severely impacted.

\section{How is the proposed BIM ecosystem approach different?}

This section briefly discusses how the proposed approach is different to the existing approaches in BIM research and practice from a systemic point of view:

Normative models of BIM research versus co-evolutionary view of BIM ecosystem. Most of the current approaches to present a systemic view around BIM are descriptive and normative. Typically, these approaches outline sequential and linear stages and directions of BIM development [15]. For example, BIM-levels 0-3 have been defined, as progressive and sequential levels. While such clear definitions of the levels are useful in determining the maturity levels and creating a roadmap, it can be argued that such an approach fails to sufficiently account for emergent trends and directions of development within the context of BIM research. For example, parallel developments such as laser scanning, internet of things (IoT), etc are being integrated into the BIM systems, but these cannot be directly straightjacketed into the current definitions of these pre-defined levels, which are limiting, and close-ended. In contrast, the ecosystem approach emphasizes a co-evolutionary view, whereby the constituent elements are mutually evolving. At the same time, it starts with a premise that the boundary of the ecosystem is fuzzy and ill-defined. Theoretically, the co-evolutionary nature and the fuzzy boundary of the ecosystem means that we do not yet understand the lifespan of the BIM ecosystem, and nor do we know where all, in which directions, and by how much, the BIM ecosystem could grow. Consequently, while assessing the maturity in BIM research is useful, the maturity models need to be revised every once in a while and the limitations of defining the maturity level at any given time need to be outlined, because the reference lifespan and scope of BIM research is inadequately understood.

The concept BIM ecosystem impact analysis or any equivalent concept does not exist. The existing approaches have (1) somewhat focused on understanding and mapping the constituent elements of what we describe as the BIM ecosystem, and the dependencies between them, i.e., kind of retrospective analysis, or (2) somewhat focused on understanding how to bring about a desired change within the context of the BIM 
ecosystem, i.e., kind of feasibility analysis. For example, developing mapping frameworks, maturity models, creating roadmaps, forecast models, etc fall in this category, with the objective to try and understand what is out there, and what can be done in the future to reach a desired state. However, the concept of impact analysis has not been considered at all, and there has been no discussion on whether the short-term or long-term implications of decisions taken in the current context can be or should be evaluated upfront. The objective of such impact analysis is to reinforce and validate the suitability of the feasible actions and solutions in the larger social, economic and environmental context, as well as to prepare in advance to address likely unintended effects of an action or solution.

Some of the other key factors that we propose to consider while developing an understanding of the BIM ecosystem, and which need clarification include:

Distinguishing between deterministic, predictive, and exploratory preparations. Developing models of a complex system with the view to try and understand likely future trends is often viewed with skepticism, because a number of forecast models and roadmaps have failed to make any meaningful contribution and they have been way off the mark. Therefore, it is important to clearly outline the purpose and expectations from a BIM ecosystem model. Rather than trying to build a deterministic or predictive model that can forecast how the BIM ecosystem will evolve, the BIM ecosystem research should allow exploration of various what-if scenarios that indicate numerous potential paths of evolution of the BIM ecosystem, given different conditions and emergent situations. It is expected that a what-if study will allow the ecosystem stakeholders to be better prepared for the likely scenarios than otherwise.

Multiplex networks. The use of social network analysis and similar methods to understand the dynamics of construction sector is not new. Several aspects of construction sector, ranging from teams and project management to understanding the industry level interaction have been studied using the network approach. Nonetheless, given the complexity of network research and the challenges in mapping the network dependencies, the typical research is limited to one view of the network, whereas the actors within a given network may have different dependencies within the same network, or may be part of multiple networks at the same time, which all might influence their decisions and actions in any given network. This is typically overlooked because multiple networks are not juxtaposed and investigated at the same time. Therefore, the multiplex networks approach [16] needs to be considered in developing a comprehensive understanding of the BIM ecosystem.

BIM is beyond tools and processes. Following considerable debate on whether BIM is merely a tool or a process, there is an increasing acceptance that BIM is a set of tools, process and policies. Nonetheless, if the research in BIM needs to be advanced it is desirable to look beyond this view as well. For example, the evolution of BIM has many similarities with Management Information Systems (MIS), where similar debates ensued for a while. Eventually MIS emerged as a formal discipline in itself with several 
notable theoretical perspectives associated with MIS research in areas such as cognition [17], media [18], social science [19], and behaviour [20]. In contrast, there has been limited theory building attempts in BIM research. Thus, it is argued that analogous to MIS we also need to view BIM as a discipline where fundamental theoretical contributions can be made both within and across multi-disciplinary boundaries.

\section{Building a comprehensive view of the BIM ecosystem}

This section reviews some of the complementary research methods and approaches that can be used to understand and analyze the BIM ecosystem.

Empirical studies. Qualitative empirical research based on case studies, surveys, workshops, interviews and similar data collection techniques is the most dominant research method in current research on building a holistic view of BIM. Typically the findings are used to identify various (success) factors associated with BIM and how they impact adoption, maturity, etc. Many of these studies focus on micro-ecosystem, especially based on case projects and feedback from the actors involved in the project. Other studies focused on macro-ecosystem tend to include workshops, surveys and focus groups to assess the status of BIM adoption, perception of actors and different stakeholders, role of standards, etc. However, barring a few notable articles, there is limited attempt to build a theory of BIM, and most articles report observations and findings without much articulation towards theory building.

Delphi studies. Delphi methods are based on systemic engagement and interviews with a panel of experts. Interviews are conducted in multiple iterative rounds, separately with each member of the panel. It is expected that the expert opinions of the BIM ecosystem will converge with each round. The success of Delphi studies is contingent on how quality of the chosen panel and how representative the panel is of the various facets of the BIM ecosystem.

Literature review. Literature review can be qualitative and subjective, which is typically the case, or they can be objective and quantitative, based on computational methods that can also analyze large data sets e.g. [4].

Data mining and analytics. With data mining and analytics, e.g. Big Data approach, it should be possible to conduct a comprehensive retrospective analysis, BIM-ERA, to assess and understand how the BIM ecosystem has evolved. The data-driven, fact-based findings can complement the views and opinions of experts about the evolution of BIM ecosystem, including those in Delphi Studies. The data analytics approach can be applied at both macro and micro levels. At macro levels, the trends and patterns can be studied at the industry level, while at the micro-level the large amount of data generated during a project or several projects can be analyzed to identify the patterns and dependencies at the project level. Furthermore, at the project level there is also a possibility now to mine all the BIM data generated during the project, and such as an 
analysis could also give insights into design decision making and associated dependencies.

Complex systems and networks. BIM ecosystems can be viewed as complex systems and networks. The complex systems approach has been applied by researchers in understanding construction projects as well the construction sector. The different related research methodologies can also be applied to the understanding of the BIM ecosystem. Some of relevant methodologies could include network analysis, mathematical and stochastic modeling, and simulations.

Simulation-based what-if studies. One of the objectives of trying to understand and build a theory of the BIM ecosystem is to be able to assess the patterns of evolution, and be able to conduct feasibility analysis as well as impact analysis. The challenges in conducting feasibility analysis and impact analysis is to be able trace the various dependencies and their consequences over a longitudinal chain. That is, an undesirable potential impact may be associated with a factor further down the value chain, and not necessarily easy to trace without a thorough analysis. As an analogy, a single move in a game of chess may be detrimental further down the game, but may not be obvious in the immediate few steps. The implications of this step is not independent of how the next few steps pan out, and how the opponent responds to the steps taken by the first player. These sequence of steps are typically non-deterministic, and hence, various possible paths of evolution are possible. Computational simulations based on probabilistic models provide a powerful method to conduct what-if studies, and these methods have been used successfully in various fields of complex systems research.

As discussed in this section, there are multiple research methods that can be used to study the BIM ecosystem, and the different methods can mutually complement and reinforce the understanding of the ecosystem, Figure 1.

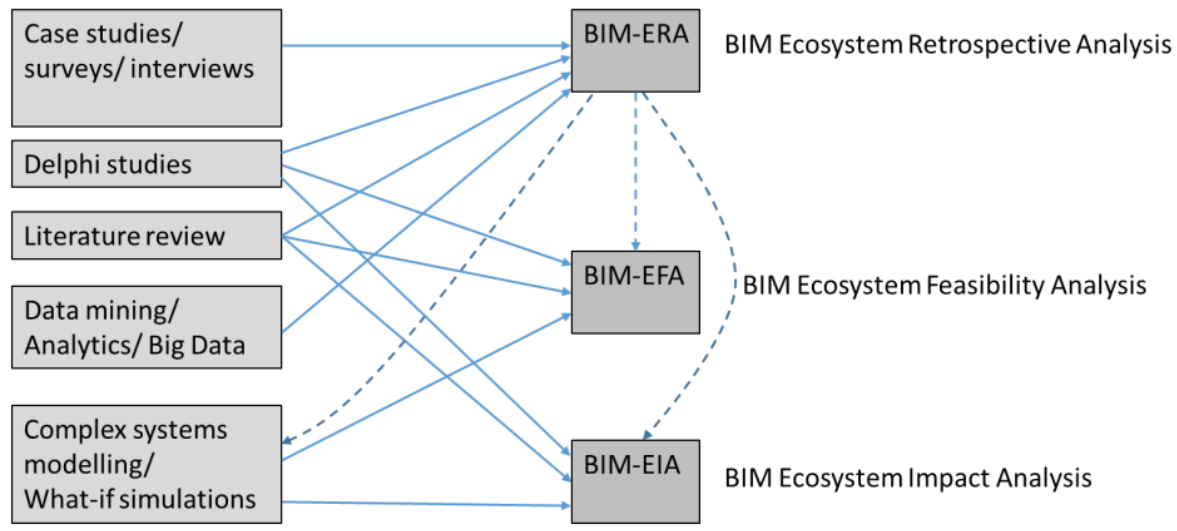

Fig. 1. Research methods applicable to different aspects of BIM ecosystem anaylsis 


\section{Conclusions}

This paper argues the need for a BIM ecosystem perspective, based on the concepts of co-evolution and emergence. The paper argues that barring a handful of notable articles there has been limited effort at building a holistic and theoretical view of BIM research environment. Furthermore, the typical approach in developing a systemic view of BIM research and development has been descriptive or normative, giving guidelines and defining levels of maturity, with limited space to account for emergent factors. The paper argues that the normative models of BIM research are limiting because they assume closed boundaries and scope of BIM. In contrast, the broader socio-technical trends such as building automation systems, IoT, big data analytics, etc are also finding their way into the mainstream BIM research and BIM systems. Consequently, discussions on maturity models and BIM levels appear hasty and misplaced until the BIM ecosystem boundaries are established with greater research rigour. Considering the co-evolutionary nature of the constituents of the BIM ecosystem, the definition of the BIM ecosystem boundary remains a challenging tasks.

Therefore, this research emphasizes the need for theoretical and methodological approaches to support BIM ecosystem analysis based on the underlying dependencies, patterns and mechanisms of co-evolution. Three different aspects of ecosystem analysis are differentiated (1) BIM-ERA that uses varied methods including qualitative studies, Delphi studies, literature review and big data analytics to review how the BIM ecosystem has evolved, based on the experience so far, (2) BIM-EFA that uses the understanding of the BIM ecosystem built from BIM-ERA, together with Delphi studies, literature review and what-if simulations based on complex systems modelling to assess the feasibility of desired actions in a given ecosystem, and (3) BIM-EIA that also uses the understanding of the BIM ecosystem built from BIM-ERA, together with Delphi studies, literature review and what-if simulations based on complex systems modelling to assess the short-term and long-term impact of a potential feasible action. One of key contributions of this paper is to highlight the need for a BIM-EIA framework, which has not been considered at all in the current BIM literature.

In addition, it must be noted that most of the current articles directed towards a holistic view of BIM research are based on expert opinions, theoretical arguments or other qualitative studies, but there is very little use of complementary methods such as complex systems modelling, what-if simulations or data analytics. It can be argued that the ability to compute emergent patterns of evolution and emergence over longitudinal patterns is limited in such qualitative approaches, while computational methods can be used to explore unlimited number of potential paths of evolution for extended periods of time, under varied what-if conditions. The usability of such computational models are dependent on how well we understand the underlying mechanisms and patterns in BIM ecosystem, and how well we are able to translate them into computational models where complex patterns emerge at global levels, based on well-established dependencies and rules of local interactions between constituent elements.

In summary, this paper argues the need for an ecosystem view of BIM research, based on diverse theoretical and methodological approaches. The paper is a first attempt at articulating the directions and approaches that are required to create such a systemic 
view, and this remains the primary limitation of the paper. Such an attempt requires collective debate, review and iterations, and one of the main purposes of this paper is exactly to initiate such a theoretical and methodological culture in BIM research..

\section{References}

1. Moore J. F.: The Death of Competition: Leadership and Strategy in the Age of Business Ecosystems. HarperBusiness, ISBN 0-88730-850-3 (1996)

2. Gu N., Singh V., London K.: BIM Ecosystem: The Coevolution of Products, Processes, and People. In: Kensek, K., Noble, D (Eds.), BIM in Current and Future Practice. pp. 197-211, (2014)

3. Bernstein H. M. (Ed): The Business Value of BIM in North America: Multi-year Trend Analysis and User Ratings (2007-2012). SmartMarket Report, McGraw-Hill, (2012).

4. Yalcinkaya M., Singh V.: Patterns and trends in Building Information Modeling (BIM) research: A Latent Semantic Analysis. Automation in Construction 59: 68-80, (2015).

5. Singh V., Gu N., Wang X.: A theoretical framework for BIM-based multi-disciplinary collaboration platform. Automation in Construction 20:134-144, (2011).

6. Succar B.: Building information modelling framework: A research and delivery foundation for industry stakeholders. Automation in Construction 18(3): 357-375, (2009).

7. Messner J., Anumba C., Dubler C., Goddman S., Kasprzak C., Kreider R., Leicht R., Saluja C., Zikic N.: BIM Project Execution Planning Guide, Penn State, (2010).

8. Succar B., Kassem M.: Macro-BIM adoption: Conceptual structures." Automation in Construction 57: 64-79, (2015).

9. Rezgui, Y., Zarli, A.: Paving the Way to the Vision of Digital Construction: A Strategic Roadmap. Journal of Construction Eng. and Manag., 132:7(767), 767-776, (2006).

10. Kim C., Kim H., Han S.H., Kim C., Kim M.K., Park S.H.: Developing a technology roadmap for construction R\&D through interdisciplinary research efforts. Automation in Construction 18(3):330-337, (2009).

11. Peters, D.P.C., Pielke R.A., Bestelmeyer B.T., Allen C.D,. Munson-McGee S., Havstad K.M.: Cross-scale interactions, nonlinearities, and forecasting catastrophic events. Proc. Natl. Acad. Sci., 101(42), 15,130-15,135. (2004)

12. Rammel C., Stagl S., Wilfing H.: Managing complex adaptive systems-a coevolutionary perspective on natural resource management. Ecological Economics 63(1):9-21, (2007).

13. Strogatz S.H.: Exploring complex networks. Nature 410: 268-276, (2011)

14. Stewart-Oaten A., Murdoch W.M., Parker K.R.: Environmental Impact Assessment: Pseudoreplication in Time? Ecology 67:929-940, (1986).

15. BIS/Industry Working Group: Building Information Modelling (BIM) Working Party Strategy Paper. Government Construction Client Group, London, (2011).

16. Kim J.Y., Goh K-I: Coevolution and Correlated Multiplexity in Multiplex Networks. Physical Review Letters 111: 058702, (2013).

17. Vessey I., Galletta D.: Cognitive fit: An empirical study of information acquisition, Information Systems Research 2: 63-84, (1991).

18. Daft R.L., Lengel R.H.: Information richness: a new approach to managerial behavior and organizational design. In: Cummings, L L and Staw, B M (Eds.), Research in organizational behavior 6: 191-233. Homewood, IL: JAI Press, (1984).

19. Mumford E.: Sociotechnical Systems Design: Evolving Theory and Practice, Manchester Business School, ISSN 0954-7401, (1985).

20. Venkatesh V., Morris M.G., Davis G.B., Davis F.D.: User Acceptance of Information Technology: Toward a Unified View. MIS Quarterly 27(3): 425-478, (2003). 